\title{
Negations and natural sampling in data selection: Ecological versus heuristic explanations of matching bias
}

\author{
MIKE OAKSFORD and MAREK MOUSSAKOWSKI \\ Cardiff University, Cardiff, Wales
}

\begin{abstract}
Matching bias occurs when people ignore negations when testing a hypothesis-for example, if $A$, then not 2-and select possible data types that are named in the hypothesis (i.e., A and 2; Evans \& Lynch, 1973). There are two explanations of this bias: the heuristic account and the contrast class account. The latter is part of Oaksford and Chater's (1994) ecological approach to data selection. On this account, a contrast set (i.e., birds that are not ravens) has a higher probability than the original set (i.e., birds that are ravens). This article reports two experiments in which these accounts make divergent predictions. The same materials were used as those in Yama (2001), who found more support for the heuristic approach. Experiment 1 replicated Yama with Western participants. Experiment 2 used a procedure introduced by Oaksford and Wakefield (2003). Rather than present participants with one of each of the four possible data types all at once, 50 were presented one at a time. The proportions of each data type reflected the relevant probabilities. The results supported the ecological approach, showing that people constructed contrast sets that strongly influenced their data selection behavior. The results were not consistent with the heuristic approach.
\end{abstract}

Since the discovery of matching bias (e.g., Evans \& Lynch, 1973), it has featured prominently in discussions of rationality, because it suggests that people may be illogical. Matching bias has been observed mainly in the Wason (1968) selection task, in which participants are presented with four cards, each with a number on one side and a letter on the other. A conditional rule is also presented-for example, if there is an A on one side then there is a 2 on the other side. The four cards show an "A," a "K," a " 2 ," and a "7." Participants select the cards to turn over to determine whether the rule is true or false. Logically, only the A and the 7 cards should be selectedthat is, those cards that may reveal a falsifying instance. However, as few as $4 \%$ of participants make this response, other responses being far more common (A and 2 cards, 46\%; A card only, 33\%; A, 2, and 7 cards, 7\%; A and 7 cards, 4\%; Johnson-Laird \& Wason, 1970).

Matching bias is revealed when negations are introduced into the task rule. For the rule if $A$ then not 2, participants select more logically correct cards - that is, those that correspond to the true antecedent (TA) case and the false consequent (FC) case. For this rule, these cases correspond to the $\mathrm{A}$ and the 2 cards, respectively. Selecting these cards for both the unnegated and negated rules is consistent with ignoring the negation and just matching the terms mentioned in the rules to the cards.

We thank Jonathan Evans, Hiroshi Yama, and an anonymous reviewer for their very helpful comments on an earlier version of this article. Correspondence concerning this article should be addressed to M. Oaksford, School of Psychology, Cardiff University, P. O. Box 901, Cardiff, CF10 3YG, Wales (e-mail: oaksford@cardiff.ac.uk).
This is matching bias, which seems to be irrational - that is, people are not attending to the logical structure of the task, but to superficial perceptual features that are irrelevant to the inferences they should draw.

There are two competing explanations of the matching bias effect (Evans, 1998): the heuristic account (Evans, 1998, 2002; Evans \& Lynch, 1973) and the contrast class account (Oaksford, 2002; Oaksford \& Chater, 1994; Oaksford \& Stenning, 1992). The goal of the work we report here was to test these accounts of matching bias in an experiment in which they make divergent predictions. We first will introduce each theory and then will locate these models in the current debate between the heuristics and biases (e.g., Tversky \& Kahneman, 1974, 1981) approach and the ecological approach to cognitive biases (e.g., Anderson, 1990; Anderson \& Sheu, 1995; Gigerenzer, Hoffrage, \& Kleinbölting, 1991; Klayman \& Brown, 1993; Klayman \& Ha, 1987; McKenzie, in press; McKenzie \& Mikkelsen, in press; Oaksford \& Chater, 1994, 1996).

\section{The Heuristic Account}

If participants are matching and ignoring the negations, it would seem that either their behavior is irrational or they are simply not engaging in the task at all. However, Evans $(1983,1984,1989,1998)$ has always denied this, arguing instead that in the heuristic-analytic theory, this behavior is caused by the pragmatic function of negations. More recently, it has become clear that matching does not mean enhanced processing of matching cases but, rather, reduced processing of mismatching cases (Evans, Clibbens, \& Rood, 1996). Nonetheless, the theoretical explanation for matching has not changed - people match 
because of linguistic heuristics indicating the irrelevance of mismatching cases (Evans, 1983, 1984, 1989, 1998). In Evans's heuristic-analytic theory, analytic (i.e., logical) reasoning processes come into play only after materials have been assessed for relevance by these heuristic processes. Wason (1965) suggested that negations are typically used to deny presuppositions. For example, "I did not go for a walk" denies the presupposition that one went for a walk. The topic of this sentence-what the sentence is about - is walking and not any of the things one could have done while not walking. The not-heuristic (Oaksford \& Stenning, 1992) treats information about-for example, what one did while not walking - as irrelevant. Attention to information not mentioned in the rule is, therefore, reduced.

\section{Contrast Classes and Optimal Data Selection}

The contrast class account provides an alternative explanation of this behavior, which suggests that negations define high-probability contrast sets. For example, the set of drinks that are not coffee is larger than the set of drinks that are coffee. According to the normative theory of optimal data selection (Oaksford \& Chater, 1994, 1996, 2003), people are highly sensitive to the probabilities of the antecedent and the consequent of a conditional hypothesis. The reason for this can be seen intuitively by considering a familiar example from the philosophy of science: If it is a raven then it is black. This rule is falsified by finding a nonblack raven but is confirmed in part by finding any other possibility - that is, a black raven, a black nonraven, or a nonblack nonraven. Of course, the most common category is nonblack nonravens: Most things are not black and are not ravens - that is, ravens and black things are rare. This means that finding a black raven or a nonblack raven is very informative about the truth or falsity of the hypothesis. Finding a black nonraven is moderately informative. But finding a nonblack nonraven is very uninformative. So, if you look at a black thing ( 2 card), you may find that it is a raven, which is very informative, or that it is a nonraven, which is moderately informative. Conversely, if you look at a nonblack thing (not-2 card), you may find that it is a raven, which is very informative, or that it is a nonraven, which is very uninformative. On average, this means that looking at black things is more informative than looking at nonblack things - that is, it is more informative to look at the true consequent (TC) card.

This intuition is formalized in optimal data selection theory and explains why in the standard selection task, participants select the A and the 2 cards: The 2 card is more informative than the not-2 card. In the normal environment, in which properties are rare, the TC card is the most informative for a purely affirmative rule - that is, one with no negations (see, also, McKenzie, Ferreira, Mikkelsen, McDermott, \& Skrable, 2001; McKenzie \& Mikkelsen, 2000).

Now consider a negated rule-for example, if it is a raven then it is not white. Using exactly the same ratio- nale, let us consider the FC case - that is, a white thing. If we look at this case, we will find either that it is a raven, which is very informative, or that it is not a raven, which is moderately informative. If we look at a nonwhite thing, we will find either that it is a raven, which is very informative, or that it is not a raven, which is very uninformative (again, because most things are not white and are not ravens). This reverses the average informativeness, so that now the FC case ( 2 card) is most informative. Consequently, people should also select the 2 card when given the if $A$ then not 2 rule to test. Note that according to this account, the matching response is not the product of linguistic heuristics, but of a normative theory of how people should select data in this task.

\section{Approaches to Cognitive Biases}

Over the last 10-20 years or so, there has been a shift in our understanding of the cognitive biases observed in human judgment, decision making, and hypothesis testing (see McKenzie, in press, for an excellent historical overview). In the heuristics and biases program (e.g., Tversky \& Kahneman, 1974), cognitive biases were understood to arise from a variety of heuristic processes, none of which was guaranteed to provide the correct answers in a domain (e.g., Tversky \& Kahneman, 1974). The heuristic explanation of matching bias falls within this framework. However, this heuristic derives from the way negations are normally interpreted - that is, in what one might call their normal linguistic environment. This fact may suggest that the heuristic approach is rational (but see below) because it relates to aspects of a more recent approach in which the contrast class and optimal data selection accounts are embedded.

More recently, the ecological approach has been used to argue that a range of cognitive biases are only apparent and arise because researchers have applied the wrong normative theory, failed to realize that people's behavior is adaptive to the normal environment, or both (again, see McKenzie, in press, for a review). Matching bias was an early target for this new approach (Oaksford \& Chater, 1994). It was argued that confirmation bias in the standard selection task could be explained by a different normative theory, optimal data selection, plus the assumption that, in people's normal environment, most properties are rare. This analysis showed that people's behavior need not be viewed as the product of heuristic processes but was an adaptive response to the normal environment. Moreover, on the contrast class account, the fact that people's behavior changed in response to varying negations in the rules showed that people's behavior was also adaptable to manipulations indicating that the normal environment had changed. Consistent with the contrast class explanation, several experiments have now shown that people's behavior changes in response to varying probabilities in the same way as it does when negations are varied (Oaksford, Chater, \& Grainger, 1999; Oaksford \& Wakefield, 2003). However, these experiments have provided only indirect evidence against the heuris- 
tic approach, since negations were not used and, so, matching bias could not be detected.

Only very recently have the heuristic and the contrast class accounts been investigated in experiments in which they made divergent predictions (Yama, 2001). However, Yama found conflicting evidence. In most of his experiments, he found evidence for both the heuristic account and the contrast class account. However, particularly in his Experiment 4 (see below), the evidence for the heuristic account was far stronger than that for the contrast class account. In the experiment we will report, we attempted to show that Yama's results were a product of a weak probability manipulation and that when a stronger manipulation is used, the contrast class account dominates. We first will provide some further reasons to question the heuristic account. We then will present Yama's experimental procedure, before outlining the probability manipulation (also used by Oaksford \& Wakefield, 2003) that we used in the main experiment, based on natural sampling (Gigerenzer \& Hoffrage, 1995). Before we present this experiment, we will report the results of a replication of Yama with Western students, carried out because Yama's participants were Japanese and there are well-documented differences in reasoning behavior between Oriental and Western participants (Peng \& Nisbett, 1999) that were relevant to the present experimental hypotheses.

\section{The Normal Function of Negations}

Both the heuristic and the contrast class accounts appeal to the normal function of negations in discourse. The heuristic account suggests that the named constituent remains the focus of attention when it is negated. In contrast, the contrast class account suggests that the relevant contrast set becomes the focus of attention. As we observed in the last section, this aspect of the heuristic account may give it the appearance of an adaptively rational strategy that appeals to normal environmental conditions, since the claim is that in the normal linguistic context of interpretation, negations usually retain the named constituent as the focus of attention, because they are normally used to deny presuppositions. Oaksford (2002) provided two reasons to suspect that there are problems with this apparently rational justification.

First, in experimental studies of negations in normal discourse, it appears that the activation of the representations of a negated constituent are suppressed - that is, they are attended to less, not more (MacDonald \& Just, 1989). Second, despite the appeal to the normal function of negations in discourse, as Evans (1998) has observed, matching is suppressed by the use of realistic contents. That is, when contents other than abstract alphanumeric stimuli are used, matching disappears. As Oaksford (2002) observed, this is not consistent with the claim that matching results from the named constituent's remaining the focus of attention in normal discourse. This hypothesis must surely predict that matching should also occur with real-world material. Indeed, one might even expect matching to be more preva- lent. After all, these are the materials from which people must learn the normal discourse function of negations. So there are some good empirical and conceptual reasons to doubt the rationale behind the heuristic account.

\section{Yama (2001)}

We now will outline Yama's (2001) Experiment 4, which provided the most support for the heuristic account, and will outline the predictions made by each theory. In that experiment, participants were told that while preparing students' health certificates, which indicated a student's blood type on one side $(\mathrm{Rh}+$ or $\mathrm{Rh}-)$, a doctor placed an $\mathrm{X}$ or a $\mathrm{Y}$ on the other side. They were also told that only $0.5 \%$ of the population were $\mathrm{Rh}-$. The participants then had to check the health certificate to see whether the doctor had been using a particular rule to determine whether an $\mathrm{X}$ or a $\mathrm{Y}$ was to be written on the certificate. Four cards representing the certificates showed, $\mathrm{X}, \mathrm{Y}, \mathrm{Rh}-$, and $\mathrm{Rh}+$. Because negations were used in the rules, we will describe the cards in terms of whether they make the antecedent or consequent true or false. For example, the TC case for the rule (1) if $X$ then $R h-$ is $\mathrm{Rh}-$; however, for the rule (2) if $X$ then not $R h-$, it is $\mathrm{Rh}+$. The FC case is $\mathrm{Rh}+$ for (1) and $\mathrm{Rh}-$ for (2). For consistency, we also will describe the $\mathrm{X}$ card as the TA case and the $Y$ card as the false antecedent (FA) case. Four rules varied whether a negation was in the consequent and whether the probability of the TC case $[P(\mathrm{TC})]$ was low or high: affirmative-low $P(\mathrm{TC})$, if there is an $\mathrm{X}$ on one side then $\mathrm{Rh}-$; affirmative-high $P(\mathrm{TC})$, if there is an $\mathrm{X}$ on one side then $\mathrm{Rh}+$; negative-low $P(\mathrm{TC})$, if there is an $\mathrm{X}$ on one side then not $\mathrm{Rh}+$; and negative-high $P(\mathrm{TC})$, if there is an $\mathrm{X}$ on one side then not $\mathrm{Rh}-$.

According to the contrast class account, participants should always select the card corresponding to the lowprobability category. Consequently, they should select more FC cards than TC cards for the high-probability rules. So for the affirmative-high and the negative-high rules, the $\mathrm{Rh}-$ card should be selected more often than the $\mathrm{Rh}+$ card. Moreover, more TC than FC cards should be selected for the low-probability rules. So for the affirmative-low and the negative-low rule, the $\mathrm{Rh}-$ card should again be selected more often than the $\mathrm{Rh}+$ card.

In contrast, according to the heuristic account, participants should always select the card that matches the case mentioned in the rule, regardless of whether it is negated. Consequently, participants should select more FC than TC cards for the negated rules. So for the negative-low rule, more $\mathrm{Rh}+$ than $\mathrm{Rh}-$ cards should be selected, and for the negative-high rule, more $\mathrm{Rh}-$ than $\mathrm{Rh}+$ cards should be selected. Moreover, more TC than FC cards should be selected for the affirmative rules. So for the affirmative-low rule, more $\mathrm{Rh}-$ than $\mathrm{Rh}+$ cards should be selected, and for the affirmative-high rule, more Rh+ than $\mathrm{Rh}-$ cards should be selected. Yama's (2001) Experiment 4 strongly confirmed the matching predictions but not the contrast class predictions. 


\section{Natural Sampling and the Sequential Selection Task}

We suggest that Yama's (2001) failure to observe the behavior predicted by the contrast class account occurred because of the weakness of his probability manipulation. Oaksford and Wakefield (2003) argued that previous probability manipulations (e.g., Green \& Over, 1997, 2000; Oaksford et al., 1999; Oberauer, Wilhelm, \& Rosas Díaz, 1999) did not capture the sequential nature of evidence gathering in the real world (but see Oaksford, Chater, Grainger, \& Larkin, 1997). This is what Gigerenzer and Hoffrage (1995) refer to as natural samplingthat is, in the real world, people update their beliefs about repeatable events by viewing each data point one at a time. During the sampling process, the probabilities of the antecedent $[P(\mathrm{TA})]$ and the consequent $[P(\mathrm{TC})]$ will be learned simultaneously. This process was also used to motivate the idea that an effective probability manipulation requires expressing probabilities as frequencies (e.g., 1 out of 10), rather than as single-event probabilities (e.g., .1, or $10 \%$ ).

Oaksford and Wakefield (2003) used both modes of presentation, and we used the same procedure here. Their experiment had three phases. The first two were similar to the procedure used by Oberauer et al. (1999). In Phase 1, the participants were first informed about the probabilities of the various cards (As, Ks, 2s, and 7s) in a pack of 1,000 cards, using frequency formats (Gigerenzer \& Hoffrage, 1995) - for example, they might be told that out of 1,000 cards, 100 had an A on one side. They then provided estimates of these probabilities to check that they had understood this information.

In Phase 2, the participants performed a sequential version of the selection task, in which they saw 40 cards one at a time. The proportion of different cards reflected the probability manipulation. So for a low-probability antecedent and consequent if $A$ then 2 rule $[P(\mathrm{TA})=.1$, $P(\mathrm{TC})=.2]$, the participants saw $2 \mathrm{As}, 42 \mathrm{~s}, 18 \mathrm{Ks}$, and $167 \mathrm{~s}$, presented one at a time in random order. They were also told that whether the card was placed with the letter or the number uppermost was randomly determined (so they should expect to see an equal amount of letters and numbers). For each card, they had to indicate whether they wanted to look at it to decide whether the rule was true or false.

Finally, in Phase 3, they classified 50 cards into the card types (A2, A7, K2, or K7) to provide indirect estimates of the probabilities of the antecedent and the consequent. These joint probabilities also allowed us to calculate conditional probabilities - in particular, $P(\mathrm{FC} \mid \mathrm{TA})$, which is a parameter of the optimal data selection model. $\mathrm{Ob}$ taining these indirect estimates was done in order to compare people's probability estimates before and after the selection task, to see whether they were different. This was because Hattori (2002) and Oaksford and Chater (2003) suggested that people's actual probabilities probably distort the given probabilities by overestimating low probabilities and underestimating high probabilities. In contradiction to Oberauer et al.'s (1999) findings, Oaksford and Wakefield's (2003) experiment produced all the effects predicted by the probabilistic approach.

However, Oaksford and Wakefield (2003) did not use rules containing negated constituents and, so, could not provide a test of the contrast class and heuristic accounts of the matching bias effect. The purpose of the present research was, therefore, to test the contrast set and heuristic accounts, using Yama's (2001) materials but using Oaksford and Wakefield's procedure.

\section{Cross-Cultural Considerations}

Before performing such an experiment, however, it was important to confirm that Yama's (2001) findings would replicate in the population in which we tested the contrast class and heuristic accounts of the matching bias effect, because Yama's experiments were conducted in Japan with Japanese students, and there is evidence of reasoning differences between Western and Oriental cultures (e.g., Peng \& Nisbett, 1999). In particular, it has been suggested that in Chinese culture, people may be less willing to decontextualize materials in order to see the underlying logical relations. Decontextualizing involves attending to statements without considering the "larger field of facts and theories in which [they] are embedded" (Peng \& Nisbett, 1999, p. 742).

This process may be particularly relevant to Yama's (2001) study, because Evans (2002) has suggested that the probabilistic effects Yama observed may occur because of subtle pragmatic effects that allow participants to contextualize a problem. If in Oriental cultures it is harder to decontextualize, probabilistic effects should be stronger in Yama's study than with Western participants. Of course, in replicating Yama's Experiment 4, the opposite concern was in focus, since only very weak probabilistic effects were observed-that is, despite their propensity to contextualize, Yama's participants apparently did not do so in this experiment. Moreover, looking for probabilistic effects with Western participants by using Yama's materials makes a strong test of the contrast class account, because Western participants are less likely to contextualize than Japanese participants. However, the compulsion to contextualize is strong across cultures and has been labeled by Stanovich (1999), "the fundamental computational bias." Thus, although they may be weaker, we would still expect to see probabilistic effects with Western participants.

\section{EXPERIMENT 1}

Experiment 1 was a direct replication of Yama's (2001) Experiment 4, using U.K. students rather than Japanese students as participants. The only variation was that the participants were told that only $12 \%$ of the participants had the Rh-blood type in the U.K. This was done in order to match the probability manipulation on this experiment to that used in Experiment 2, where we directly tested the contrast class and the heuristic accounts. If we 
had kept to the $0.5 \%$ figure used by Yama, we would have had to use a very large number of trials to achieve the probability manipulation in Experiment 2.

\section{Method}

Participants. One hundred undergraduate psychology students from Cardiff University took part in return for course credit. All the participants came from the U.K. or other European countries. None had any prior knowledge of the selection task.

Design. The experiment was a $4 \times 2 \times 2$ mixed design with card (TA vs. FA vs. TC vs. FC) as a within-subjects factor and $P(\mathrm{TC})$ (high vs. low) and negation (affirmative vs. negative) as betweensubjects factors. The two between-subjects factors defined the four experimental conditions - that is, affirmative-low, affirmative-high, negative-low, and negative-high.

Materials and Procedure. The experiment was presented on a single sheet of paper to groups of participants ranging in size from 2 to 5 people. The participants were randomly assigned to one of the four experimental conditions, giving 25 participants in each. The affirmative-low condition will be used to illustrate the instructions and the procedure. The participants were first thanked for agreeing to take part and were told that the whole procedure should last only a few minutes at most. They were asked to take their time to read the instructions carefully before answering the question. They then read the following instructions:

I want you to imagine that Dr. A has to prepare health certificate cards for students at the University. A student's blood type, $\mathrm{Rh}+$ or $\mathrm{Rh}-$, is printed on one side of a card and Dr. A has put an X or a Y on the other side for every card. There are 1,000 cards. 120 of the cards have $\mathrm{Rh}-$ and 880 have $\mathrm{Rh}+$ on one side, reflecting the low $12 \%$ incidence of this blood type in the UK population.

I want you to check whether Dr. A assigned Xs and Ys so that:

"If there is an $\mathrm{X}$ on one side of a card, then $\mathrm{Rh}-$ is on the other side."

To do this I want you to imagine that four cards have been drawn at random one at a time from the pile of 1,000 cards. The four cards are placed in front of you with only one side showing and your task is to say whether you want to turn the card over to help decide whether Dr. A assigned letters according to the above rule. Do not turn cards unnecessarily.

The four cards, showing $\mathrm{Rh}-, \mathrm{Rh}+, \mathrm{X}$, and $\mathrm{Y}$, were given below these instructions. In the affirmative-high condition, the rule was if there is an $X$ on one side of a card, then $R h+i$ on the other side. In the negative-low condition, it was if there is an $X$ one side of a card, then $R h+i$ not on the other side. In the negative-high condition, it was if there is an X on one side of a card, then Rh-is not on the other side. The participants were told that if they wanted to turn a card, they should tick it unambiguously. They also were told that once they had finished, they should tell the experimenter, who thanked them and debriefed them about the purpose of the experiment.
It could be argued that asking the participants to turn the cards they "want" to turn, versus turning the cards they must turn, may have biased them to interpret the task inductively, rather than deductively. However, as Oaksford and Wakefield (2003) observed, participants were also told, "Do not turn cards unnecessarily"- that is, turn only cards it is necessary to turn, or turn only the cards they must turn. Consequently, the use of "want" could not bias people away from a deductive interpretation of the task.

\section{Results and Discussion}

In this experiment, 2 participants spoiled their answer sheets, and their data were therefore excluded. This meant that the two low-probability conditions had 24, rather than 25, participants in them. The results of Experiment 1 are shown in Table 1, which also shows the results of Yama's (2001) Experiment 4. In a log-linear analysis, Yama found a highly significant main effect of negation for the FC card, so that more were selected when a rule was negative than when it was affirmative, because it now matched the item named in the rule. Experiment 1 strongly replicated this effect $\left[G^{2}(1, N=98)=10.89, p<.001 ; G^{2}\right.$ is the log-likelihood ratio that is distributed approximately as $\chi^{2}$. Yama also found a main effect of probability in the predicted direction, which we did not replicate $\left[G^{2}(1, N=98)=0.03\right.$, n.s.]. However, we did find a significant interaction, such that the effect of negation was found only when the $P(\mathrm{TC})$ was high $\left[G^{2}(1, N=\right.$ $50)=10.34, p<.005]$, but not when it was low $\left[G^{2}(1\right.$, $N=48)=2.13$, n.s.]. For the other cards, Yama (2001) found no other significant main effects or interactions. However, we found significant main effects for the TC card for both negations $\left[G^{2}(1, N=98)=5.31, p<.025\right]$ and probabilities $\left[G^{2}(1, N=98)=4.16, p<.05\right]$, both in the predicted direction. That is, there were more TC card selections when the rules were affirmative than when they were negative and when $P(\mathrm{TC})$ was low rather than high.

Despite some differences, these results mirrored those found by Yama (2001). In particular, the effects of negations were stronger than the effects of probabilities. To further confirm that these data replicated those in Yama, we performed $\chi^{2}$ goodness-of-fit tests for each card, using the proportion of each card selected for each rule in Yama as the predicted probability for each cell. For all

Table 1

Proportions of Each Card Selected in Each Condition of Experiment 1, Showing the Corresponding Proportions in Yama's (2001) Experiment 4

\begin{tabular}{|c|c|c|c|c|c|c|c|c|}
\hline \multirow[b]{3}{*}{ Condition } & \multicolumn{8}{|c|}{ Card } \\
\hline & \multicolumn{2}{|c|}{ TA } & \multicolumn{2}{|c|}{ FA } & \multicolumn{2}{|c|}{$\mathrm{TC}$} & \multicolumn{2}{|c|}{$\mathrm{FC}$} \\
\hline & Ex. & Yama & Ex. & Yama & Ex. & Yama & Ex. & Yama \\
\hline $\mathrm{AL}$ & .83 & .73 & .13 & .25 & .58 & .40 & .33 & .21 \\
\hline $\mathrm{AH}$ & .88 & .85 & .28 & .15 & .40 & .37 & .20 & .37 \\
\hline NL & .79 & .74 & .17 & .13 & .38 & .34 & .54 & .53 \\
\hline $\mathrm{NH}$ & .76 & .77 & .16 & .13 & .16 & .21 & .64 & .66 \\
\hline
\end{tabular}

Note-Ex., results of Experiment 1; Yama, results of Yama's Experiment 4; AL, affirmative-low rule; $\mathrm{AH}$, affirmative-high rule; NL, negative-low rule; $\mathrm{NH}$, negative-high rule; TA, true antecedent card; FA, false antecedent card; TC, true consequent card; FC, false consequent card. 
the cards, the hypothesis that our results simply replicated Yama's could not be rejected [TA, $\chi^{2}(3, N=98)=$ $1.91, p>.10$; FA, $\chi^{2}(3, N=98)=5.78, p>.10$; TC, $\chi^{2}(3, N=98)=4.09, p>.10 ; \mathrm{FC}, \chi^{2}(3, N=98)=$ $5.33, p>.10$ ]. In sum, Yama's findings were replicated with the Western students that we then used to test the hypothesis that probabilistic effects will be observed when natural sampling is used to administer the probability manipulation.

\section{EXPERIMENT 2}

In this experiment, we used Yama's (2001) materials with the sequential selection task procedure introduced by Oaksford and Wakefield (2003). There have been previous attempts to allow participants to learn the relevant probabilities in a selection task with negated rules (Pollard \& Evans, 1983). Moreover, the results seemed to contradict Oaksford and Chater's $(1994,1996)$ optimal data selection theory and the contrast class account. In Pollard and Evans, participants learned that the $P(\mathrm{TC})$ of the rule if $A$ then 2 was .64. They also performed a selection task with the rule if $A$ then not 2 , for which the $P(\mathrm{TC})$ was, therefore, .36. That is, one condition provided an affirmative-high rule, and the other provided a negative-low rule. The participants selected more FC cards and fewer TC cards for the negative-low rule than for the affirmative-high rule. Although consistent with the heuristic account, this result is not consistent with the contrast class account (Evans \& Over, 1996).

Pollard and Evans's (1983) results have never been replicated. However, Yama's (2001) four experiments and our Experiment 1 provided the same conditions, albeit without the learning phase. These results were not consistent with Pollard and Evans. In a meta-analysis treating experiment as the unit of analysis (Glass, McGaw, \& Smith, 1981), there were no differences between the affirmativehigh rules $(M=.44, S D=.19)$ and the negative-low rules $(M=.55, S D=.02)$ in the proportion of FC cards selected $[t(4)=1.30, p=.26]$. This was also true for the TC cards [affirmative-high, $M=.41, S D=.10$; negativelow, $M=.49, S D=.12 ; t(4)=1.21, p=.32]$. The trend for the FC card was in the right direction according to the heuristic approach. However, for the TC card, the trend was in the direction predicted by the contrast class account.

The explanation for this discrepancy may be that Pollard and Evans's (1983) learning procedure provided a stronger probability manipulation than did those in Yama (2001) and in Experiment 1. However, this does not seem consistent with the participants' then ignoring the probability manipulation and matching more strongly in Pollard and Evans. Nonetheless, it may be that the effect occurs only when the probability manipulation is strong, in which case our natural sampling procedure provides a strong test of the heuristic versus contrast class accounts.

Oaksford and Chater (1998) proposed an alternative explanation for Pollard and Evans's (1983) findings. Pol- lard and Evans used a prediction task for the learning phase, in which participants must predict what is on the other side of a card, which is then revealed to them. Oaksford and Chater (1998) argued that participants focus attention on learning the conditional probabilities of what is on the other side of the cards, since this is what the task demands. Knowing these conditional probabilities does not constrain the values of the probabilities of the antecedent and the consequent. Therefore, meeting the learning criterion in the learning task is no indication that people have learned the latter probabilities. Moreover, the participants did not provide estimates of the probabilities of the antecedent and the consequent in Pollard and Evans's experiments. Consequently, it was quite consistent for the participants to assume their normal default rarity values for these probabilities.

Oaksford and Wakefield's (2003) procedure does not use a prediction task and, so, is not subject to a similar criticism. Moreover, in their procedure, subjective estimates are obtained of all the relevant probabilities at two points during the task, so there is no need to infer that these probabilities have been learned. However, Oaksford and Wakefield's procedure does potentially introduce some confounds, which it is important to identify and avoid.

The contrast class account predicts that participants should select the same card in each condition: the rarely occurring Rh - card. However, because this card occurs rarely, higher proportions might be selected without the participants interpreting the rules according to the contrast class account. If the participants select some of each type of card at random and then stop, they will always select a higher proportion of $\mathrm{Rh}-$ than $\mathrm{Rh}+$ cards. So, in this experiment, if they select two of each, this would amount to $67 \% \mathrm{Rh}-$ selections but only $9 \% \mathrm{Rh}+$ selections. But this need have nothing to do with the way they interpret the rules. Oaksford and Wakefield (2003) identified this possibility and showed how it could be ruled out by considering the frequency of first and last cards selected. Selecting at random and then stopping predicts that card selections should fall to zero by the last card selected and certainly should never rise. In the Results and Discussion section, we will present a similar analysis for this experiment.

Nonetheless, people may still be ignoring the rules. However, if participants interpret the rules correctly in Phase 1, traces of these interpretations should be observable in Phase 3. In Phase 1, the participants are given information about the probability of the consequent and must provide ratings of the probabilities of the antecedent and the consequent. In Phase 3 , they provide information about the same probabilities after having performed the selection task. The expected effects arise from the different strategies that may be adopted when the probability of the antecedent is greater than the probability of the consequent. The participants are not given any information about the distribution of $\mathrm{X}$ and $\mathrm{Y}$. So all they know is that $\mathrm{X}$ and $\mathrm{Y}$ are members of a binary ad hoc category 
(Barsalou, 1983): "things Dr. A writes on cards.” Oaksford (2002) argued that, "when the material is truly binary, it seems that the default may be to make an equiprobability assumption" (p. 142). This is because the rarity assumption cannot apply - that is, $\mathrm{X}$ and $\mathrm{Y}$ cannot both be rare. Therefore, the probabilities of $\mathrm{X}$ and $\mathrm{Y}$ should be around .5. If this is the case, then for the low-probability rules (affirmative-low and negative-low), $P(\mathrm{TA})$ is greater than $P(\mathrm{TC})$. But this will be apparent to the participants only if they interpret the negations according to the contrast class account. Moreover, this means that these rules must have many counterexamples, because there must be more cards with an $\mathrm{X}$ on them than with $\mathrm{Rh}-$.

Only two strategies have been proposed for how participants deal with being asked to test a rule that they know has many counterexamples. First, they may revise down $P$ (TA) (Oaksford \& Chater, 1994). This would predict that in Phase 3, $P(\mathrm{TA})$ should be lower for the lowprobability $[P(\mathrm{TC})]$ rules. Second, participants may assume that these rules allow many counterexamples, while nonetheless being predictively useful (Oaksford \& Wakefield, 2003). That is, using the affirmative-low rule as an example, even though there are many exceptions, there is a higher probability of someone being $\mathrm{Rh}-$, given that $\mathrm{X}$ is written on the card, than would be expected by chance. This would predict that the probability of exceptions - that is, the probability of an FC given a TA $[P(\mathrm{FC} \mid \mathrm{TA})]$ - should be higher for the low-probability rules. Of course, both strategies may be in evidence. To preempt, in the Results and Discussion section, we also will show how such changes in these probabilities directly predict some effects observed for the antecedent cards in Phase 2.

In sum, there were several predictions tested in this experiment. First, in the sequential selection task (Phase 2), according to the contrast class account, the participants should select more FC than TC cards for the highprobability rules and more TC than FC cards for the lowprobability rules. The heuristic account predicts that the participants should select more FC than TC cards for the negated rules and more TC than FC cards for the affirmative rules. Second, by providing probability information in Phases 1 and 2 and obtaining probability ratings, we were also able to test that the participants were interpreting the rules as predicted and that these interpretations survived to influence the participants' probability judgments after they had performed the selection task.

\section{Method}

Participants. Eighty undergraduate psychology students from Cardiff University took part in return for course credit. None had any prior knowledge of the selection task.

Design. The design of the main selection task phase of the experiment (Phase 2) was the same as that in Experiment 1.

Materials and Procedure. The experiment was presented on a Macintosh IIsi computer using the PsyScope software (Cohen, MacWhinney, Flatt, \& Provost, 1993) to control the presentation of stimuli and to record responses. The participants were randomly assigned to one of the four experimental conditions, giving 20 participants in each. The affirmative-low condition will be used to illus- trate the instructions and the procedure. The participants first read the following instructions. All the instructions were self-paced, and the space bar had to be pressed to initiate the next stage of the experiment. The participants were first thanked for taking part and were told the approximate duration of the experiment and that it would contain three phases. Upon a press of the space bar, the experimental situation was displayed. This was identical to the first paragraph of the instructions in Experiment 1. Upon a press of the space bar, the instructions for the first phase of the experiment were presented. The instructions read as follows:

I now want you to imagine that a single card is drawn from the pack of a thousand cards. What I would like you to do is rate how likely it is on a scale from 0 to 9 that this card has an $\mathrm{X}$, a Y, an $\mathrm{Rh}-$ or an $\mathrm{Rh}+$ on it. You will be prompted for each judgement and you should use the number keys at the top of the keyboard to respond.

Upon pressing the space bar, the participants were presented with four statements one at a time in random order. Each statement was of the form, "Please rate how likely it is that the card has an $\mathrm{Rh}-$ on it," one for each card type (X, Y, Rh-, and Rh+). On completing this phase of the experiment, the instructions for the second phase were displayed. These instructions were same as those for Experiment 1 after the first paragraph but also included instructions specifically for the computer presentation. In particular, they were told that they would see each card one at a time as it was drawn from the pack of 1,000 cards. They were also told the following:

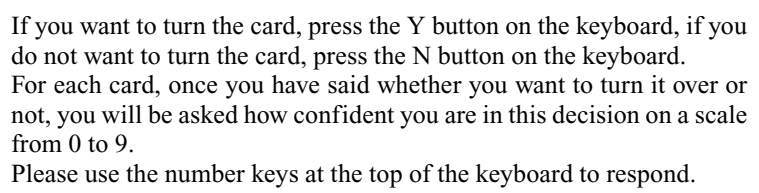

The rules were the same as those in Experiment 1. We also collected confidence ratings and reaction times (RTs) for each card selection. Upon pressing the space bar, the participants were then presented with 50 cards in random order, two showing an X, 23 showing a Y, 3 showing $\mathrm{Rh}-$, and 22 showing $\mathrm{Rh}+$. These were in direct proportion to the probability information that the participants had already been given. There were 25 cards showing X or $\mathrm{Y}$ and 25 cards showing $\mathrm{Rh}-$ or $\mathrm{Rh}+$. Which card was seen on each trial was determined randomly from the 50 cards without replacement.

Upon completing this phase, the instructions for the final phase of the experiment were displayed:

In this final phase of the experiment I want you to imagine that I have placed all the cards back into the pack. I now randomly pick cards out of the pack, BUT you are NOT allowed to see either side of the card. What I want you to do is assume that the rule is true and then for each card I pick out randomly from the pack, indicate whether you think:

It has an $\mathrm{X}$ on one side and $\mathrm{Rh}+$ on the other side $\mathrm{X}, \mathrm{Rh}+($ Press Key 1$)$, or

It has an $\mathrm{Rh}-$ on one side and an $\mathrm{X}$ on the other side $\mathrm{Rh}-, \mathrm{X}$ (Press Key 2), or

It has an $\mathrm{Y}$ on one side and an $\mathrm{Rh}-$ on the other side $\mathrm{Y}, \mathrm{Rh}-$ (Press Key 3), or

It has an $\mathrm{Rh}+$ on one side and a Y on the other side Rh+, Y (Press Key 4)

(There are no other possibilities.)

Each time I pick a card out you will be prompted: NEW CARD!

Upon pressing the space bar, the participants were then prompted 50 times to respond to a "new card" being drawn from the pack. They were asked to assume that the rule was true.

\section{Results and Discussion}

Although we recorded confidence ratings and RTs, we will not report these data, since they do not add to the analysis of selection frequencies. As in Oaksford and 
Table 2

Direct (Phase 1) and Indirect (Phase 3) Estimates of the Probabilities of the True Antecedent (TA), the False Antecedent (FA), the True Consequent (TC), and the False Consequent (FC) in Each Condition in Experiment 2

\begin{tabular}{|c|c|c|c|c|c|c|c|c|c|c|c|c|c|}
\hline \multirow[b]{3}{*}{ Estimate } & \multirow[b]{3}{*}{ Condition } & \multicolumn{12}{|c|}{ Probability Assessed } \\
\hline & & \multicolumn{3}{|c|}{$P(\mathrm{TA})$} & \multicolumn{3}{|c|}{$P($ FA $)$} & \multicolumn{3}{|c|}{$P(\mathrm{TC})$} & \multicolumn{3}{|c|}{$P(\mathrm{FC})$} \\
\hline & & $M$ & $S D$ & Med. & $M$ & $S D$ & Med. & $M$ & $S D$ & Med. & $M$ & $S D$ & Med. \\
\hline \multirow[t]{4}{*}{ Direct } & $\mathrm{AL}$ & .48 & .25 & .5 & .51 & .24 & .5 & .17 & .24 & .1 & .91 & .11 & .9 \\
\hline & $\mathrm{AH}$ & .44 & .17 & .5 & .53 & .13 & .5 & .77 & .29 & .9 & .13 & .19 & .1 \\
\hline & NL & .48 & .17 & .5 & .44 & .14 & .5 & .18 & .18 & .1 & .69 & .31 & .9 \\
\hline & NH & .49 & .25 & .5 & .50 & .20 & .5 & .78 & .26 & .9 & .11 & .19 & .0 \\
\hline \multirow[t]{4}{*}{ Indirect } & $\mathrm{AL}$ & .34 & .18 & .4 & .66 & .18 & .6 & .24 & .15 & .2 & .76 & .15 & .8 \\
\hline & $\mathrm{AH}$ & .53 & .27 & .5 & .47 & .27 & .5 & .73 & .22 & .7 & .27 & .22 & .3 \\
\hline & NL & .47 & .14 & .5 & .53 & .14 & .5 & .34 & .18 & .4 & .66 & .18 & .6 \\
\hline & NH & .48 & .27 & .5 & .52 & .27 & .5 & .63 & .23 & .7 & .37 & .23 & .3 \\
\hline
\end{tabular}

Note-AL, affirmative-low rule; AH, affirmative-high rule; NL, negative-low rule; NH, negative-high rule; Med., median value to one decimal place.

Wakefield (2003), confidence ratings and selection frequencies were highly correlated, and RTs were longer for cards with a high proportion of selections.

Probability estimates. Table 2 shows the probability assessments when measured directly in Phase 1 and measured indirectly in Phase 3. Although the participants were given a $0-9$ scale, it was clear from the data that very few participants had used the 0 value (1.25\% of the responses). We therefore treated the scale as ranging from 1-9 and transformed the ratings to a $0-1$ scale by taking away one and dividing by eight. Although the relation between the participants' actual subjective probabilities and these rescaled ratings can be questioned, we assumed that they provided a reasonably accurate reflection.

The median values of the probabilities (to one decimal place) mirrored the given probabilities - that is, $\operatorname{Prob}$ (TC) was .1 for the affirmative-low and negativelow rules and .9 for the affirmative-high and negativehigh rules (we used Prob to distinguish judged probabilities from given probabilities). Given the rating scale, this was about as accurate as the participants could be (recall that the participants were informed that $12 \%$ of the U.K. population were $\mathrm{Rh}-$ ). Moreover, for the negated rules, these results show that they were interpreting the probabilities of the negated categories pretty accurately as the complements of the affirmative cases. Prob(TA) was .5 , confirming Oaksford's (2002) conjecture that people make an equiprobability assumption for truly binary materials. As we suggested, this means that the participants must have realized that $P(\mathrm{TA})$ was greater than $P(\mathrm{TC})$ for the low-probability rules. That these initial ratings reflected the probability manipulation replicated Oaksford and Wakefield (2003). However, in that experiment, the participants did not have to interpret negations, and they were provided with information about $P(\mathrm{TA})$, as well as about $P(\mathrm{TC})$. So they did not have to make an equiprobability assumption for $P(\mathrm{TA})$.

For $P(\mathrm{TA})$, Table 2 shows that people's subjective estimates in Phase 3 were not altered much by performing the sequential selection task in Phase 2-that is, the me- dian values (to one decimal place) were still .5 or close. The participants initially assumed that $\mathrm{X}$ and $\mathrm{Y}$ were equiprobably distributed in a sample of 1,000 cardsthat is, 500 are $\mathrm{X}$ and 500 are Y. By natural sampling, the small number of cards they observed in Phase 2 would not be expected to alter these subjective estimates by much $[(475+2) / 1,000=.477]$. For $P(\mathrm{TC})$, when the Phase 3 probabilities were low $(<.5)$, they were higher than in Phase 1. Moreover, when they were high in Phase 3 $(>.5)$, they were lower than in Phase 1. This is consistent with the participants' subjective probabilities in Phase 3, underestimating high probabilities and overestimating low probabilities. This possibility has been proposed as a result of model-fitting exercises (Hattori, 2002; Oaksford \& Chater, 2003) and also was confirmed experimentally by Oaksford and Wakefield (2003). This finding need not imply any cognitive bias, only that the ratings in Phase 1 and the probabilities collected in Phase 3 are not perfectly correlated, which results in a regression toward the mean. Thus, this effect may simply be a reflection of error variance in a regressive environment (Budescu, Wallsten, \& Au, 1997; Dawes \& Mulford, 1996; Erev, Wallsten, \& Budescu, 1994; Juslin, Winman, \& Olsson, 2000).

Card selections. Table 3 shows the mean proportions of each card selected in Phase 2 of the experiment, in which the participants carried out the sequential selection task and learned about the probabilities by natural sampling. We carried out a $2 \times 2 \times 2$ mixed analysis of variance (ANOVA) with $P(\mathrm{TC})$ (high vs. low) and negation (affirmative vs. negative) as between-subjects factors and card (TC vs. FC) as a within-subjects factor. According to the contrast class account, there should be a two-way interaction between probability and card, so that the proportion of FC cards selected is higher than that of TC cards when $P(\mathrm{TC})$ is high and the proportion of TC cards selected is higher than that of FC cards when $P(\mathrm{TC})$ is low. According to the heuristic account, there should be a two-way interaction between negation and card, so that the proportion of FC cards selected is higher 
Table 3

Mean Proportions (and Standard Deviations) of Each Card Selected in Each Condition in Experiment 2

\begin{tabular}{|c|c|c|c|c|c|c|c|c|}
\hline \multirow[b]{3}{*}{ Condition } & \multicolumn{8}{|c|}{ Card } \\
\hline & \multicolumn{2}{|c|}{ TA } & \multicolumn{2}{|c|}{ FA } & \multicolumn{2}{|c|}{$\mathrm{TC}$} & \multicolumn{2}{|c|}{$\mathrm{FC}$} \\
\hline & $M$ & $S D$ & $M$ & $S D$ & $M$ & $S D$ & $M$ & $S D$ \\
\hline $\mathrm{AL}$ & .70 & .41 & .20 & .28 & .67 & .39 & .32 & .36 \\
\hline $\mathrm{AH}$ & .60 & .42 & .27 & .26 & .42 & .37 & .45 & .33 \\
\hline NL & .50 & .40 & .40 & .26 & .53 & .36 & .40 & .33 \\
\hline $\mathrm{NH}$ & .38 & .46 & .26 & .28 & .33 & .33 & .57 & .43 \\
\hline
\end{tabular}

Note-AL, affirmative-low rule; AH, affirmative-high rule; NL, negative-low rule; $\mathrm{NH}$, negative-high rule; TA, true antecedent card; FA, false antecedent card; TC, true consequent card; FC, false consequent card.

than that of TC cards when the consequent is negated and the proportion of TC cards selected is higher than that of FC cards when the consequent is affirmative.

All the main effects and the three-way interaction failed to reach significance [in each case, $F(1,76)<1$ ]. The two-way interaction between negation and card was not significant $\left[F(1,76)=2.90, M S_{\mathrm{e}}=0.152, p=.09\right]$, although the trends were in the right direction. There was a higher proportion of FC cards $(M=.48, S D=.38)$ selected than TC cards $(M=.43, S D=.36)$ when the consequent was negated. Moreover, there was a higher proportion of TC cards $(M=.54, S D=.40)$ selected than FC cards $(M=.39, S D=.35)$ when the consequent was affirmative.

The only significant effect was the two-way interaction between probability and card $[F(1,76)=8.95$, $\left.M S_{\mathrm{e}}=0.152, p<.005\right]$. In simple effects comparisons, there was a higher proportion of TC cards $(M=.60$, $S D=.38)$ selected than FC cards $(M=.36, S D=.33)$ when $P\left(\right.$ TC) was low $\left[F(1,76)=7.22, M S_{\mathrm{e}}=0.133, p<\right.$ $.01]$. Moreover, there was a higher proportion of $\mathrm{FC}$ cards $(M=.51, S D=.39)$ selected than TC cards $(M=$ $.38, S D=.35)$ when $P(\mathrm{TC})$ was high, although this just failed to reach significance $\left[F(1,76)=3.47, M S_{\mathrm{e}}=\right.$ $0.130, p=.066]$.

We also performed separate between-subjects ANOVAs by card, replicating the analyses performed by Yama (2001) and in Experiment 1. For the TC card, there was a main effect of probability $\left[F(1,76)=7.22, M S_{\mathrm{e}}=\right.$ $0.133, p<.01]$, no main effect of negation $[F(1,76)=$ $\left.1.95, M S_{\mathrm{e}}=0.133, p=.17\right]$, and no interaction $[F(1,76)<$ 1]. The proportion of TC cards selected was higher when $P(\mathrm{TC})$ was low than when it was high. For the FC card, there was a close-to-significant main effect of probability $\left[F(1,76)=3.45, M S_{\mathrm{e}}=0.130, p=.067\right]$, no main effect of negation $\left[F(1,76)=1.42, M S_{\mathrm{e}}=0.130, p=.24\right]$, and no interaction $[F(1,76)<1]$. The proportion of FC cards selected was higher when $P(\mathrm{TC})$ was high than when it was low. These results confirmed the predictions of the contrast class account, but not those of the heuristic account.

These results were not due to a superficial strategy such as selecting a few of each card type at random and then stopping. This strategy would predict that the par- ticipants' card selections should be high initially and then fall to zero (Oaksford \& Wakefield, 2003). However, in paired sign tests across conditions, there were no significant differences in the probability of selecting a consequent card between the first and the last encounters $[\mathrm{Rh}+($ first $=.50$, last $=.39), p=.11 ; \mathrm{Rh}-($ first $=$ .53 , last $=.59), p=.47]$. Card selections never fell to zero and, indeed, rose for $\mathrm{Rh}-$. This result also replicated within conditions. These results are not consistent with this superficial processing strategy.

Table 3 reveals that negations would appear to have influenced antecedent card selections. In an ANOVA similar to that for the consequent cards, the only significant effect was a two-way interaction between negation and card $\left[F(1,76)=5.81, M S_{\mathrm{e}}=0.160, p<.025\right]$. There was a higher proportion of TA cards $(M=.60, S D=.38)$ selected than FA cards $(M=.36, S D=.33)$ when the rule was affirmative $\left[F(1,76)=21.37, M S_{\mathrm{e}}=0.160\right.$, $p<.0001]$. Moreover, the proportion of TA cards selected was higher when the rule was affirmative than when it was negative $\left[F(1,76)=5.11, M S_{\mathrm{e}}=0.177, p<\right.$ $.05]$. Similar effects were also observed by Yama (2001), although not in his Experiment 4. Oaksford (2002) suggested that negations in the consequent might be affecting selection of antecedent cards by influencing the probabilities of the antecedent. As we now will argue, these effects for the antecedent cards are a direct result of participants' having to deal with testing hypotheses that they know allow many exceptions - that is, for low-probability rules. We broached this possibility in the introduction, and we now will show how these effects have been revealed in the data.

Probabilistic interpretation. In the introduction, we observed that if participants were interpreting the rules according to the contrast class account, either the $P(\mathrm{TA})$ should be lower, or the probability of exceptions, $P(\mathrm{FC} \mid \mathrm{TA})$, should be higher, for the low-probability rules. Both of these effects were observed. We first conducted a $2 \times 2$ ANOVA with probability $[P(\mathrm{TC})]$ and negations as factors and the $P(\mathrm{TA})$ estimates in Phase 3 as the dependent variable (see Table 2 ). $P($ TA) was significantly lower when $P(\mathrm{TC})$ was low $(M=.41, S D=.16)$ than when it was high $\left[M=.51, S D=.27 ; F(1,76)=6.66, M S_{\mathrm{e}}=\right.$ $0.049, p<.025]$. There was no main effect of negation and no significant interaction $[F(1,76)<1$ in both cases]. In a similar ANOVA, $P(\mathrm{FC} \mid \mathrm{TA})$ was significantly higher when $P(\mathrm{TC})$ was low $(M=.57, S D=.32)$ than when it was high $\left[M=.37, S D=.28 ; F(1,78)=10.19, M S_{\mathrm{e}}=\right.$ $0.084, p<.0025]$. There was also a significant effect of negation, so that $P(\mathrm{FC} \mid \mathrm{TA})$ was significantly higher when the rule was negated $(M=.56, S D=.29)$ than when it was affirmed $\left[M=.38, S D=.32 ; F(1,78)=7.10, M S_{\mathrm{e}}=\right.$ $0.084, p<.01]$. Although the negative-low rule did not show as great a reduction in $P(\mathrm{TA})$ as the affirmative-low rule, the participants compensated by increasing $P(\mathrm{FC} \mid \mathrm{TA})$, which was the highest for this rule at .62 .

These results could be predicted only if the participants interpreted the rules in Phase 1, including the negations, according to the contrast class account. Recall that the 
participants saw the rules only once, during Phase 1 . Consequently, these interpretations survived the selection task phase to affect Phase 3. They must, therefore, have been in play during Phase 2. Moreover, these results show how the manipulation of negations in the consequent can affect antecedent card selections by influencing $P(\mathrm{TA})$. This seems to confirm Oaksford's (2002) conjecture about the similar effects observed by Yama (2001). We now will show that these changes in $P(\mathrm{TA})$ could directly predict the selections of the TA card in Phase 2.

Subjective probabilities in the mid-range are likely to be accurate - that is, they should be the least affected by regression toward the mean. Consequently, we would expect the values of $P(\mathrm{TA})$ found in Phase 3 to accurately reflect those used in data selection in Phase 2. The Phase 3 probability estimates should, therefore, provide good estimates of the best-fit values of the $P(\mathrm{TA})$ parameter of Oaksford and Chater's $(1994,1996,1998,2003)$ optimal data selection model when it is fitted to the Phase 2 data on card selection frequencies. We therefore fitted the model to the data from Phase 2 of the experiment (see Table 4). ${ }^{1}$ The overall fit was good $\left(R^{2}=.82\right.$, RMS error $=$ $.061)$. Moreover, the best-fit values for $P(\mathrm{TA})$ were very close to the Phase 3 estimates [see Table 2; affirmativelow, $P(\mathrm{TC})=.38$; affirmative-high, $P(\mathrm{TC})=.43$; negative-low, $P(\mathrm{TC})=.48$; negative-high, $P(\mathrm{TC})=$ .47]. A $\chi^{2}$ goodness-of-fit test showed that the hypothesis that the observed values of $P(\mathrm{TC})$ in Phase 3 were the same as the predicted best-fit values based on the Phase 2 card selection frequencies could not be rejected $\left[\chi^{2}(3)=\right.$ $4.84, p>.10]$.

In sum, these results seem to be consistent with only one interpretation. Although $P(\mathrm{TA})$ was not explicitly manipulated, the effects observed for the antecedent cards in Phase 2 were a direct consequence of the participants' interpreting the rules probabilistically, in accordance with the contrast class account of negations and the theory of optimal data selection. Given that there were no negations in the antecedents of these rules, the heuristic account provides no explanation of the observed effects for the antecedent cards. Oaksford (2002) made a similar observation regarding the very similar effects observed by Yama (2001).

Table 4

Predicted Proportions of Card Selections in Experiment 2 Based on Oaksford and Chater's Optimal Data Selection Model

\begin{tabular}{ccccc}
\hline & \multicolumn{4}{c}{ Card } \\
\cline { 2 - 5 } Condition & TA & FA & TC & FC \\
\hline AL & .72 & .25 & .66 & .28 \\
AH & .65 & .33 & .45 & .48 \\
NL & .52 & .42 & .54 & .41 \\
NH & .52 & .41 & .35 & .62 \\
\hline
\end{tabular}

Note-AL, affirmative-low rule; AH, affirmative-high rule; NL, negative-low rule; $\mathrm{NH}$, negative-high rule; TA, true antecedent card; FA, false antecedent card; TC, true consequent card; FC, false consequent card.

\section{GENERAL DISCUSSION}

These experiments confirm that by using a stronger probability manipulation in the sequential selection task, the behavior predicted by the contrast class account and the optimal data selection model dominate. In Experiment 1, we showed that Yama's (2001) results do replicate with Western participants.

In Experiment 2, not only were the participants told about the probability manipulation, as in Yama (2001), but also they experienced the probabilities during the course of performing the sequential selection task. In this experiment, although there were highly significant effects of the probability manipulation, the effects of negations were far weaker. As in Oaksford and Wakefield (2003), it was also possible to demonstrate that card selections were not simply a function of a nonrational random strategy. Such a strategy is also inconsistent with the interpretational effects we observed. Because people must make an equiprobability assumption for the antecedent cards, the low $P(\mathrm{TC})$ rules must have many exceptions. According to optimal data selection, this requires that they revise either $P(\mathrm{TA})$ down or $P(\mathrm{FC} \mid \mathrm{TC})$ up. In their Phase 3 probability estimates, it appeared that the participants did both. Moreover, the probabilities of $P($ TA $)$ in Phase 3 were almost identical to the best-fitting values when the optimal data selection model was fitted to the results of the sequential selection task in Phase 2. Consequently, the participants were not responding randomly but in accordance with a probabilistic interpretation of the rules.

The latter results are also not consistent with the proposal that people simply recode the negations with these binary materials (Evans et al., 1996) - that is, they recode if $X$ then not $R h+$ as if $X$ then $R h-$. This might explain the effects for the negated rules, but it cannot account for the probabilistic effects observed for the purely affirmative rules. Nor can it account for previous findings showing probabilistic effects when negations are not used (Oaksford et al., 1999; Oaksford et al., 1997; Oaksford \& Wakefield, 2003). Moreover, like the heuristic account, the recoding hypothesis offers no explanation of the interpretational effects we have just discussed. For example, recoding the negative-low rule could not explain why $P(\mathrm{FC} \mid \mathrm{TA})$ was so high for this rule in Phase 3 or why there were any effects observed for the antecedent cards in Phase 2. In contrast, the contrast class and optimal data selection accounts provide a rational explanation for all the effects we observed in Experiment 2. It is, of course, possible that nonbinary materials may lead to some of the effects predicted by the heuristic approach. This must await further experimentation. However, as Oaksford (2002) pointed out, Oaksford and Stenning's (1992) materials were not binary, but matching bias was not evident.

It may appear paradoxical that a rational account could predict the low selection rates for the TA card (see Table 3), since it may seem that turning this card is something that people should always do. The mean proportion of this 
card chosen for the high-probability rules is only .49 . However, when the probabilities of both the antecedent and the consequent are high [as they are for these rules, because of the equiprobability assumption for $P(\mathrm{TA})]$, then according to optimal data selection, the TA card should be chosen much less frequently. We can again see why this is by looking at the average informativeness for the antecedent cards for the rule if it is not black, then it is not a raven - that is, a rule for which the contrast class account predicts that the antecedent and the consequent should have a high probability. If we look at the nonblack thing (TA), we will find either that it is not a raven, which is very uninformative, or that it is a raven, which is very informative. If we look at a black thing (FA), we will find either that it is a raven, which is very informative, or that it is not a raven, which is moderately informative. That is, on average, it is more informative to look at the FA case. Similar low selection rates of the TA card for rules with a high-probability antecedent and consequent were observed by Oaksford and Wakefield (2003).

Although our results were not consistent with the heuristic account, there is other evidence supportive of this theory. Evans et al. (1996) showed that another way of removing matching was to use explicit negations on the cards: "a number that is not 2," rather than, say, "7." Thus, according to Evans et al. (1996), matching bias is produced by the need to infer that 7 is a member of the category not-2. However, participants do not respond logically in the selection task even with explicit negations. Rather, they now select the TA and TC cases even for rules containing negated antecedents and consequents. Similar behavior is observed when realistic content is used (Reich \& Ruth, 1982) and when contrast class construction is facilitated (Oaksford \& Stenning, 1992). Oaksford (2002) argued that the latter effects are consistent with people's appropriately contextualizing contrast class construction.

One of Reich and Ruth's (1982) realistic thematic content rules, if I travel to France, I do not go by plane, can be used to illustrate this point. Here, it is quite clear that the contrast class cannot consist of all possible modes of transport. Rather, it has to consist of a mode of transport suitable for traveling long distances over water. That is, a boat seems to be the most likely contrast class member (although it could be a hovercraft, a hydrofoil, or the channel tunnel). The probability of traveling by boat is obviously a lot lower than that of not traveling by plane. This explains why, for this example rule, selecting the TA case (France) and the TC case (boat) was the rational strategy. A contrast class that is easy to construct generally coincides with a category (or disjunction of categories) that has a lower probability than does the complement. That is, the crucial feature of a contrast set for an Item $\mathrm{A}\left(\mathrm{A}^{\mathrm{c}}\right)$ is that $P(\mathrm{~A}) \leq P\left(\mathrm{~A}^{\mathrm{c}}\right) \leq 1-P(\mathrm{~A})$. Quite where in this interval $P\left(\mathrm{~A}^{\mathrm{c}}\right)$ falls will depend largely on prior world knowledge.

This account of contrast class construction also generalizes to the use of explicit and implicit negations in other reasoning tasks (Oaksford, 2002; Oaksford, Chater, \& Larkin, 2000). However, it does not account for the effect of explicit negation in the selection task. Oaksford suggested that this manipulation may encourage a more extreme form of matching. As we have seen, even in the present experiment, there remains a residual tendency to match, which is probably attributable to inattention to the task. Using explicit negations may encourage people to match named items including the negation. This is what Oaksford called the matching ${ }_{2}$ strategy, and it will result in people's selecting the TA and TC cases even for rules containing negated antecedents and consequents. Consequently, although explicit negations produce the same effects as realistic materials (Reich \& Ruth, 1982) or disambiguating contrast class construction (Oaksford \& Stenning, 1992), this may not be for the same reasons. However, it must be conceded that Oaksford's matching 2 conjecture has not been tested experimentally.

Moreover, Evans et al. (1996) also showed a reduction of matching for another task, the truth table evaluation task. In this task, participants are given a rule, if $p$ then $q$, and then for each case - that is, $p, q$; $p$, not- $q$; not- $p$, $q$; and not- $p$, not- $q$ - they must evaluate whether it makes the rule true or false or is irrelevant. Here, reductions in matching for explicit negations were accompanied "by only modest increases in logical accuracy" (Evans et al., 1996, p. 404). Indeed, the increase was not significant for the standard if $p$ then $q$ rule form, but only for the logically equivalent $p$ only if $q$ rule form. Thus, there is some evidence that explicit negations may lead to increases in logical performance. However, this finding stands in clear need of more convincing experimental demonstration. Moreover, Oaksford (2002) has also provided a matching ${ }_{2}$ interpretation of this effect.

In conclusion, these results are consistent with the view that matching bias is unlikely to be the result of applying an inappropriate cognitive heuristic, as in the heuristics and biases approach. Rather, consistent with more recent ecological approaches, when negations are introduced into the selection task, people's behavior seems to display a rational and adaptable response to a perceived change in the environment, so that rarity can no longer be assumed.

\section{REFERENCES}

Anderson, J. R. (1990). The adaptive character of thought. Hillsdale, NJ: Erlbaum.

Anderson, J. R., \& Sheu, C.-F. (1995). Causal inferences as perceptual judgments. Memory \& Cognition, 23, 510-524.

Barsalou, L. W. (1983). Ad hoc categories. Memory \& Cognition, 11, 211-227.

Budescu, D. V., Wallsten, T. S., \& Au, W. T. (1997). On the importance of random error in the study of probability judgment: Part II. Applying the stochastic judgment model to detect systematic trends. Journal of Behavioral Decision Making, 10, 173-188.

Cohen, J., MacWhinney, B., Flatt, M., \& PRovost, J. (1993). PsyScope: An interactive graphic system for designing and controlling experiments in the psychology laboratory using Macintosh computers. $\underline{B e-}$ havioral Research Methods, Instruments, \& Computers, 25, 257-271. DaWEs, R. M., \& Mulford, M. (1996). The false consensus effect and 
overconfidence: Flaws in judgment or flaws in how we study judgment? Organizational Behavior \& Human Decision Processes, 65, 201-211.

Erev, I., Wallsten, T. S., \& Budescu, D. V. (1994). Simultaneous over- and underconfidence: The role of error in judgment processes. Psychological Review, 101, 519-527.

EvANS, J. S. T. (1983). Linguistic determinants of bias in conditional reasoning. Quarterly Journal of Experimental Psychology, 35A, 635-644.

Evans, J. S. T. (1984). Heuristic and analytic processes in reasoning. British Journal of Psychology, 75, 451-468.

Evans, J. S. T. (1989). Bias in human reasoning: Causes and consequences. Hillsdale, NJ: Erlbaum.

Evans, J. S. T. (1998). Matching bias in conditional reasoning: Do we understand it after 25 years? Thinking \& Reasoning, 4, 45-82.

Evans, J. S. T. (2002). Matching bias and set sizes: A discussion of Yama (2001). Thinking \& Reasoning, 8, 153-163.

Evans, J. S. T., Clibbens, J., \& Rood, B. (1996). The role of implicit and explicit negations in conditional reasoning bias. Journal of Memory \& Language, 35, 392-409.

EvANS, J. S. T., \& LYNCH, J. S. (1973). Matching bias in the selection task. British Journal of Psychology, 64, 391-397.

Evans, J. S. T., \& Over, D. E. (1996). Rationality in the selection task: Epistemic utility versus uncertainty reduction. Psychological Review, 103, 356-363.

GiGERENZER, G., \& Hoffrage, U. (1995). How to improve Bayesian reasoning without instruction: Frequency formats. Psychological Review, 102, 684-704.

GigerenZer, G., Hoffrage, U., \& Kleinbölting, H. (1991). Probabilistic mental models: A Brunswikian theory of confidence. Psychological Review, 98, 506-528.

Glass, G. V., McGaW, B., \& SMITH, M. L. (1981). Meta-analysis in social research. Beverly Hills, CA: Sage.

Green, D. W., \& Over, D. E. (1997). Causal inference, contingency tables and the selection task. Current Psychology of Cognition, 16, 459-487.

Green, D. W., \& Over, D. E. (2000). Decision theoretical effects in testing a causal conditional. Current Psychology of Cognition, 19, 51-68.

HatTori, M. (2002). A quantitative model of optimal data selection in Wason's selection task. Quarterly Journal of Experimental Psychology, 55A, 1241-1272.

JoHNSON-LAIRD, P. N., \& WASON, P. C. (1970). Insight into a logical relation. Quarterly Journal of Experimental Psychology, 22, 49-61.

Juslin, P., Winman, A., \& Olsson, H. (2000). Naïve empiricism and dogmatism in confidence research: A critical examination of the hard-easy effect. Psychological Review, 107, 384-396.

Klayman, J., \& Brown, K. (1993). Debias the environment instead of the judge: An alternative approach to reducing error in diagnostic (and other) judgment. Cognition, 49, 97-122.

KLAYMAN, J., \& HA, Y.-W. (1987). Confirmation, disconfirmation, and information in hypothesis testing. Psychological Review, 94, 211-228.

LoEHLE, C. (2000). Global Optimization 4.0 [Computer program]. Naperville, IL: Loehle Enterprises.

MaCDonald, M. C., \& Just, M. A. (1989). Changes of activation levels with negation. Journal of Experimental Psychology: Learning, Memory, \& Cognition, 15, 633-642.

MCKenZIE, C. R. M. (in press). Judgment and decision making. In K. Lamberts \& R. Goldstone (Eds.), Handbook of cognition. London: Sage.

McKenzie, C. R. M., Ferreira, V. S., Mikkelsen, L. A., McDermott, K. J., \& Skrable, R. P. (2001). Do conditional hypotheses target rare events? Organizational Behavior \& Human Decision Processes, 85, 291-309.

MCKenZie, C. R. M., \& Mikkelsen, L. A. (2000). The psychological side of Hempel's paradox of confirmation. Psychonomic Bulletin \& Review, 7, 360-366.

McKenziE, C. R. M., \& Mikkelsen, L. A. (in press). A Bayesian view of covariation assessment. Cognitive Psychology.

OAKSFORD, M. (2002). Contrast classes and matching bias as explanations of the effects of negation on conditional reasoning. Thinking \& Reasoning, 8, 135-151.

OAKSFORD, M., \& CHATER, N. (1994). A rational analysis of the selection task as optimal data selection. Psychological Review, 101, 608-631.
OAKSFORD, M., \& CHATER, N. (1996). Rational explanation of the selection task. Psychological Review, 103, 381-391.

OAKSFORD, M., \& CHATER, N. (1998). A revised rational analysis of the selection task: Exceptions and sequential sampling. In M. Oaksford \& N. Chater (Eds.), Rational models of cognition (pp. 372-398). Oxford: Oxford University Press.

OAKSFORD, M., \& CHATER, N. (2003). Optimal data selection: Revision, review, and reevaluation. Psychonomic Bulletin \& Review, 10, 289-318.

OAKsford, M., Chater, N., \& Grainger, B. (1999). Probabilistic effects in data selection. Thinking \& Reasoning, 5, 193-243.

OAKsford, M., Chater, N., Grainger, B., \& LarKin, J. (1997). Optimal data selection in the reduced array selection task (RAST). Journal of Experimental Psychology: Learning, Memory, \& Cognition, 23, 441458.

OAKsford, M., Chater, N., \& LARKIn, J. (2000). Probabilities and polarity biases in conditional inference. Journal of Experimental Psychology: Learning, Memory, \& Cognition, 26, 883-899.

OAKSFORD, M., \& STENNING, K. (1992). Reasoning with conditionals containing negated constituents. Journal of Experimental Psychology: Learning, Memory, \& Cognition, 18, 835-854.

OAKSFORD, M., \& WAKEFIELD, M. (2003). Data selection and natural sampling: Probabilities do matter. Memory \& Cognition, 31, 143-154.

Oberauer, K., Wilhelm, O., \& Rosas Díaz, R. (1999). Bayesian rationality for the Wason selection task? A test of optimal data selection theory. Thinking \& Reasoning, 5, 115-144.

Peng, K., \& NisbeTt, R. E. (1999). Cülture, dialectics, and reasoning about contradiction. American Psychologist, 54, 741-754.

Pollard, P., \& Evans, J. S. T. (1983). The effect of experimentally contrived experience on reasoning performance. Psychological Research, 45, 287-301.

REICH, S. S., \& RUTH, P. (1982). Wason's selection task: Verification, falsification and matching. British Journal of Psychology, 73, 395405.

Stanovich, K. E. (1999). Who is rational? Studies of individual differences in reasoning. Mahwah, NJ: Erlbaum.

TVERSKY, A., \& Kahneman, D. (1974). Judgment under uncertainty: Heuristics and biases. Science, 185, 1124-1131.

TVersky, A., \& Kahneman, D. (1981). The framing of decisions and the psychology of choice. Science, 211, 453-458.

Wason, P. C. (1965). The contexts of plausible denial. Journal of Verbal Learning \& Verbal Behavior, 4, 7-11.

WASON, P. C. (1968). Reasoning about a rule. Quarterly Journal of Experimental Psychology, 20, 273-281.

Wolfram, S. (1991). Mathematica [Computer program]. Reading, MA: Addison-Wesley.

YAMA, H. (2001). Matching versus optimal data selection in the Wason selection task. Thinking \& Reasoning, 7,295-311.

\section{NOTE}

1. The optimal data selection model's predictions were compared with the mean proportion of cards selected by looking for the parameter values that provided the least sum of squared differences between data and model. This was achieved using a steepest descent search implemented in Mathematica's MultiStartMin function (Loehle, 2000; Wolfram, 1991). We report two measures of fit. The coefficient of variation, $R^{2}$, indicates the proportion of variance the model explains and, therefore, how well it fits the overall trend in the data. We also report the root-mean squared (RMS) error, which is related to the average deviation between the predicted value and the observed value of each data point. The optimal data selection model has three free parameters: $P(\mathrm{TA}), P(\mathrm{TC})$, and the exceptions parameter $[P(\mathrm{FC} \mid \mathrm{TA})]$. To model these data, the exceptions parameter was set to the empirical values obtained in Phase 3. For further details of the information gain model, see Hattori (2002), Oaksford and Chater (2003), and Oaksford and Wakefield (2003).

(Manuscript received October 17, 2002; revision accepted for publication October 28, 2003.) 